\title{
Note on the role of friction-induced momentum conservation in the collisional drift wave instability
}

\author{
J. Vranjes \\ Center for Plasma Astrophysics, Celestijnenlaan 200B, 3001 Leuven, Belgium, and \\ Faculté des Sciences Appliquées, avenue F.D. Roosevelt 50, 1050 Bruxelles, Belgium \\ S. Poedts \\ Center for Plasma Astrophysics, Celestijnenlaan 200B, 3001 Leuven, Belgium, and \\ Leuven Mathematical Modeling and Computational Science Center (LMCC)
}

\begin{abstract}
The collisional drift wave instability is reexamined taking into account the ion response in the direction parallel to the magnetic field lines, which appears due to friction with electrons and which can not be omitted in view of the momentum conservation. A modified instability threshold is obtained. In plasmas with dominant electron collisions with neutrals, the instability threshold is shifted towards higher frequencies, compared to the case of dominant electron collisions with ions. The difference between the two cases vanishes when the ion sound response is negligible, i.e., when the instability threshold disappears, and both ions and neutrals react to the electron friction in the same manner.
\end{abstract}

PACS Numbers: 52.35.Kt, 52.30.Ex, 52.20.Fs 
I. Within the standard fluid theory, the drift wave can be excited by electron collisions. In this case, the usual relation between the electron perturbed density and the perturbed potential, $n_{1} / n_{0}=e \phi_{1} / \kappa T_{e}$, becomes modified due to the presence of the collisional term, so that the potential lags behind the density. ${ }^{1,2}$ The effect appears regardless whether the electrons collide, in their predominant motion along the magnetic field vector, with ions or with neutrals. ${ }^{3,4}$ Compared with the kinetic instability (which is due to the inverse electron Landau damping effect, that appears because the mode frequency is slightly below the diamagnetic frequency), the collisional instability is dominant ${ }^{5}$ provided that the electron parallel mean-free path is smaller than the parallel wavelength. Hence the interest in the drift modes with very large parallel wave-lengths and relatively short perpendicular wavelengths. In view of the early theoretical prediction ${ }^{6,7}$ and experimental verification, ${ }^{8}$ the amount of literature dealing with the drift wave is enormous. More recently, collisional drift waves have been studied experimentally in cylindric plasma in Refs. 9-11. Details on the experimental investigation of global coherent structures associated with the drift mode in simple magnetized torus can be found in recent Refs. 12-14. The presence of charged grains on the drift wave in a cylindric configuration has been studied in Ref. 15, while the role of the drift wave in the transport phenomena may be found in the most recent Refs. 16, 17.

Some convenient approximations that are made in the derivations include the limit in which the complete dynamics of the heavier particles (i.e. ions and neutrals) in the direction along the magnetic field is negligible, and the limit when electrons can be treated as inertia-less

$$
v_{T e} \gg \omega / k_{z} \gg c_{s}, \quad c_{s}=\left(\kappa T_{e} / m_{i}\right)^{1 / 2}
$$

In the presence of collisions, the friction force term in the electron parallel momentum equation is usually written in the form $-m_{e} n_{e} \nu_{e j} \vec{v}_{e}$, where $j=i, n$. As a result one obtains a standard phase shift in the electron Boltzmann distribution $n_{1} / n_{0}=\left(e \phi_{1} / \kappa T_{e}\right)(1-i \delta)$, that is responsible for the mode growth.

However, the conservation of momentum implies that the friction term in the electron momentum equation should read $-m_{e} n_{e} \nu_{e j}\left(\vec{v}_{e}-\vec{v}_{j}\right)$ even when conventional criteria for a negligible parallel dynamics of heavier particles are fulfilled, so that the corresponding momentum component of the heavier species includes the friction term $-m_{j} n_{j} \nu_{j e}\left(\vec{v}_{j}-\vec{v}_{e}\right)$. Below, we perform derivations with such a full friction force term for some simple cases in 
order to demonstrate the differences introduced by this friction-induced response of the heavier species.

II. We first discuss a plasma with dominant collisions between the charged particles. Note that this case may also include a rather weakly ionized plasma with $n_{0} \ll n_{n}$ (the index 0 here and below denotes the electron or ion equilibrium quantities). This is because of the much larger cross section for collisions between charged particles. To have dominant collisions with protons in a plasma containing electrons, protons and neutral atoms, the electron number density should satisfy the condition

$$
\frac{n_{0}}{n_{n}}>\frac{3 \sigma_{e n}\left(4 \pi \varepsilon_{0} \kappa T_{e}\right)^{2}}{(8 \pi)^{1 / 2} e^{4} L_{e i}}
$$

Here we used the standard notation, and $L_{e i}$ is the Coulomb logarithm. Taking as an example $T_{e}=10^{4} \mathrm{~K}$, which gives ${ }^{18,19} \sigma_{e n}=2.5 \cdot 10^{-19} \mathrm{~m}^{2}$ (here $L_{e i}=6$ for $n_{0}=10^{18} \mathrm{~m}^{-3}$ ), it turns out that the electron-ion collisions are more frequent than the electron-neutral collisions provided that $n_{0} / n_{n}>0.009$. For the given temperature this is close to well known estimate ${ }^{20}$ showing that, in terms of electron collisions, an ion is equivalent to $3.4 \cdot 10^{5}\left(300 / T_{e}\right)^{2} \simeq 300$ neutral atoms.

We use the continuity equation for electrons and ions placed in an external magnetic field $\vec{B}_{0}=B_{0} \vec{e}_{z}$

$$
\frac{\partial n_{j}}{\partial t}+\nabla_{\perp}\left(n_{j} \vec{v}_{\perp j}\right)+\nabla_{z}\left(n_{j} \vec{v}_{z j}\right)=0, \quad j=e, i,
$$

where the linearized perpendicular velocities of electrons and ions are given by

$$
\begin{gathered}
\vec{v}_{\perp e 1}=\frac{1}{B_{0}} \vec{e}_{z} \times \nabla_{\perp} \phi_{1}-\frac{v_{T e}^{2}}{\Omega_{e}} \vec{e}_{z} \times \frac{\nabla_{\perp} n_{e 1}}{n_{e 0}}, \quad v_{T e}^{2}=\kappa T_{e} / m_{e} \\
\vec{v}_{\perp i 1}=\frac{1}{B_{0}} \vec{e}_{z} \times \nabla_{\perp} \phi_{1}-\frac{1}{\Omega_{i} B_{0}} \frac{\partial}{\partial t} \nabla_{\perp} \phi_{1} .
\end{gathered}
$$

Here, the effects of collisions on the perpendicular electron dynamics is neglected in contrast to the parallel one, which is justified ${ }^{21}$ as long as $k_{z}^{2} \Omega_{e}^{2} /\left(k_{y}^{2} \nu_{e i}^{2}\right)>1$.

The electron parallel velocity is determined from

$$
0=e n_{0} \frac{\partial \phi_{1}}{\partial z}-\kappa T_{e} \frac{\partial n_{1}}{\partial z}-m_{e} n_{0} \nu_{e i}\left(v_{e z 1}-v_{i z 1}\right),
$$

and the ion velocity from

$$
\frac{\partial v_{i z 1}}{\partial t}=-\frac{e}{m_{i}} \frac{\partial \phi_{1}}{\partial z}-\nu_{i e}\left(v_{i z 1}-v_{e z 1}\right) .
$$


III. In what follows, we first present the 'standard' derivation in which the ion parallel velocity is only due to the parallel component of the perturbed electric field, while the friction induced term in (7) is omitted with the usual excuse of the huge difference in mass between the two species. In this case, the perturbed ion number density is described by $^{1,2}$

$$
\frac{n_{1}}{n_{0}}=\left(\frac{\omega_{*}}{\omega}+\frac{k_{z}^{2} c_{s}^{2}}{\omega^{2}}-k_{y}^{2} \rho_{s}^{2}\right) \frac{e \phi_{1}}{\kappa T_{e}} .
$$

Using Eqs. (3) , (66) the perturbed electron number density can be written as

$$
\begin{gathered}
\frac{n_{1}}{n_{0}}=\frac{\omega_{*}+k_{z}^{2} c_{s}^{2} / \omega+i k_{z}^{2} D_{z}}{\omega+i k_{z}^{2} D_{z}} \frac{e \phi_{1}}{\kappa T_{e}}, \\
D_{z}=\frac{v_{T e}^{2}}{\nu_{e i}}, \quad \omega_{*}=-\frac{k_{y} \kappa T_{e}}{e B_{0}} \frac{n_{0}^{\prime}}{n_{0}} .
\end{gathered}
$$

Here, we have taken $\nabla n_{0}=\vec{e}_{x} d n_{0} / d x$, the perturbations are assumed to be of the form $\sim f(x) \exp \left(-i \omega t+i k_{y} y+i k_{z} z\right)$, and we work in the frame of a local approximation. The full collisional term, with perpendicular electron collisions included, should read $i\left(k_{z}^{2} v_{T e}^{2} / \nu_{e i}+\rho_{e}^{2} k_{y}^{2} \nu_{e i}\right)$, though the second term here is negligible in the limit discussed earlier. Here, $\rho_{e}=v_{T e} / \Omega_{e}$ is the electron gyro-radius.

Note that $k_{z}^{2} c_{s}^{2} / \omega$ in Eq. (9), that appears due to the term $\nu_{e i} v_{i z 1}$ in Eq. (6) , is usually omitted in standard derivations [2]. The quasi-neutrality yields the dispersion equation

$$
\frac{\omega_{*}}{\omega}+\frac{k_{z}^{2} c_{s}^{2}}{\omega^{2}}-k_{y}^{2} \rho_{s}^{2}=\frac{\omega_{*}+k_{z}^{2} c_{s}^{2} / \omega+i k_{z}^{2} D_{z}}{\omega+i k_{z}^{2} D_{z}} .
$$

We now take the limit

$$
|\omega| \ll k_{z}^{2} D_{z}
$$

and assume that $\omega$ and $\omega_{*}$ are of the same order. Used for convenience ${ }^{1}$, the condition (11) is in fact not always easily satisfied. Physically it describes ${ }^{5}$ the condition of isothermal electrons along the field lines that has been assumed. It can be rewritten as $\left(\omega / k_{z}\right) / v_{T e} \ll$ $k_{z} v_{T e} / \nu_{e i}$. The right-hand side gives the ratio of the electron mean free path $v_{T e} / \nu_{e i}$ and the parallel wavelength, that in fact must be much less than unity in order to remain within a proper fluid theory (i.e., for collisions being able to maintain Maxwellian distribution).

A few comments here are noteworthy.

i) If the parallel ion dynamics is completely neglected, we can write the dispersion equation as follows: $\Delta\left(\omega, k_{y}\right) \equiv \Delta_{r}\left(\omega, k_{y}\right)+i \Delta_{i}\left(\omega, k_{y}\right) \simeq \Delta_{r}\left(\omega_{r}, k_{y}\right)+i \omega_{i} \partial \Delta_{r}\left(\omega, k_{y}\right) /\left.\partial \omega\right|_{\omega=\omega_{r}}+$ 
$i \Delta_{i}\left(\omega_{r}, k_{y}\right)=0$, where $\left|\Delta_{i}\right| \ll\left|\Delta_{r}\right|$ and where the subscripts $r, i$ denote 'real' and 'imaginary'. Setting the real and imaginary parts of this dispersion equation equal to zero yields

$$
\omega_{r} \simeq \omega_{*} /\left(1+k_{y}^{2} \rho_{s}^{2}\right), \quad \omega_{i} \simeq-\Delta_{i}\left(\omega_{r}, k_{y}\right) /\left(\partial \Delta_{r} / \partial \omega\right)_{\omega=\omega_{r}}=\frac{\omega_{*}^{2}}{k_{z}^{2} D_{z}} \frac{k_{y}^{2} \rho_{s}^{2}}{\left(1+k_{y}^{2} \rho_{s}^{2}\right)^{3}} .
$$

The increment is proportional to $\nu_{e i}$, which implies that, with the fixed ion background (in the parallel direction), the more collisions the larger the growth of the wave is. Regarding the dependence on $k_{y} \rho_{s}$, the increment is maximal at $k_{y} \rho_{s}=1 / \sqrt{2}$.

ii) With the ion sound response taken into account (assuming that $k_{z}^{2} c_{s}^{2}$ is same order or not much larger than $\omega^{2}$ and $\left.\omega_{*} \omega\right)$, the dispersion equation becomes

$$
\omega^{2}\left(1+k_{y}^{2} \rho_{s}^{2}\right)-\omega_{*} \omega-k_{z}^{2} c_{s}^{2}+\frac{i \omega}{k_{z}^{2} D_{z}}\left[\omega\left(\omega-\omega_{*}\right)-k_{z}^{2} c_{s}^{2}\right]=0 .
$$

Here, in the expansion on the right-hand side in Eq. (10), the sound contribution yields a real and an imaginary term. The former, yielding a negligible correction to the real frequency, is neglected, and we have only kept the imaginary term. The real part of the frequency is determined from

$$
\Delta_{r}\left(\omega_{r}, k_{y}\right) \equiv \omega_{r}^{2}\left(1+k_{y}^{2} \rho_{s}^{2}\right)-\omega_{*} \omega-k_{z}^{2} c_{s}^{2}=0 .
$$

This is used in the imaginary part, yielding

$$
\omega_{i} \simeq-\Delta_{i}\left(\omega_{r}, k_{y}\right) /\left(\partial \Delta_{r} / \partial \omega\right)_{\omega=\omega_{r}}=\frac{\omega_{r}^{2}}{k_{z}^{2} D_{z}} \frac{\omega_{r}^{2} k_{y}^{2} \rho_{s}^{2}}{\omega_{r}^{2}\left(1+k_{y}^{2} \rho_{s}^{2}\right)+k_{z}^{2} c_{s}^{2}} .
$$

iii) We note that in fact in the standard literature [2] the sound contribution $k_{z}^{2} c_{s}^{2} / \omega$ on the right-hand side in Eq. (10) is neglected. It is easily seen that the origin of this term is the term $m_{e} n_{0} \nu_{e i} v_{i z 1}$ in Eq. (6) . In this case instead of Eq. (15) we obtain

$$
\omega_{i} \simeq \frac{\omega_{r}^{2}}{k_{z}^{2} D_{z}} \frac{\omega_{r}^{2} k_{y}^{2} \rho_{s}^{2}-k_{z}^{2} c_{s}^{2}}{\omega_{r}^{2}\left(1+k_{y}^{2} \rho_{s}^{2}\right)+k_{z}^{2} c_{s}^{2}}
$$

Hence, there appears to be a threshold for the instability. 
IV. Of course, a self-consistent analysis should include the full friction force effect in both, i.e., the ion and electron, parallel equations. This is simply due to the fact that the two forces are necessarily equal by magnitude. Hence, we keep Eqs. (6, 7) as they are, with the complete friction terms.

Combining the two parallel momentum equations, and using the conservation of momentum $m_{i} \nu_{i e}=m_{e} \nu_{e i}$, yields $v_{i z 1}=k_{z} c_{s}^{2} n_{1} /\left(\omega n_{0}\right)$. Instead of Eq. (8) , we now have

$$
\frac{n_{1}}{n_{0}}\left(1-\frac{k_{z}^{2} c_{s}^{2}}{\omega^{2}}\right)=\left(\frac{\omega_{*}}{\omega}-k_{y}^{2} \rho_{s}^{2}\right) \frac{e \phi_{1}}{\kappa T_{e}} .
$$

A procedure similar as earlier, now yields the electron number density

$$
\frac{n_{1}}{n_{0}}=\frac{\omega_{*}+i k_{z}^{2} D_{z}}{\omega-k_{z}^{2} c_{s}^{2} / \omega+i k_{z}^{2} D_{z}} \frac{e \phi_{1}}{\kappa T_{e}},
$$

instead of Eq. (9). Compare these two equations with Eqs. (8) and (9). Notice that in both Eqs. (17) and (18), the ion parallel response appears in a completely different manner.

Within the same approximations as earlier (i.e., $\omega^{2}, \omega_{*} \omega, k_{z}^{2} c_{s}^{2} \ll k_{z}^{4} D_{z}^{2}$ ), the dispersion equation that we now have is:

$$
\omega^{2}\left(1+k_{y}^{2} \rho_{s}^{2}\right)-\omega_{*} \omega-k_{z}^{2} c_{s}^{2}+\frac{i\left(\omega^{2}-k_{z}^{2} c_{s}^{2}\right)\left(\omega^{2}-\omega_{*} \omega-k_{z}^{2} c_{s}^{2}\right)}{\omega k_{z}^{2} D_{z}}=0 .
$$

The real frequency is the same as in the earlier Eq. (14). Hence, using Eq. (14) the imaginary part of the frequency now becomes

$$
\omega_{i} \simeq \frac{\omega_{r}^{2} k_{y}^{2} \rho_{s}^{2}}{k_{z}^{2} D_{z}} \frac{\omega_{r}^{2}-k_{z}^{2} c_{s}^{2}}{\omega_{r}^{2}\left(1+k_{y}^{2} \rho_{s}^{2}\right)+k_{z}^{2} c_{s}^{2}}=\frac{\omega_{r}^{2} k_{y}^{2} \rho_{s}^{2}}{k_{z}^{2} D_{z}} \frac{\omega_{*} \omega_{r}-\omega_{r}^{2} k_{y}^{2} \rho_{s}^{2}}{\omega_{r}^{2}\left(1+k_{y}^{2} \rho_{s}^{2}\right)+k_{z}^{2} c_{s}^{2}} .
$$

We remark the obvious difference in the instability threshold in the expression (16), and the correct expression (20), and we stress the absence of the threshold in Eq. (15).

V. The ion response to the friction is usually neglected on the basis of the huge difference in mass of the different species. However, this difference in mass may be compensated by the frequent electron collisions with ions, so that sooner or later the ions start to move in the parallel direction due to the electron drag. To get a feeling on the effects of collisions and the corresponding time scales, we may discuss the following two separate cases.

a) Assuming that the condition (1) is satisfied, the ions respond in the parallel direction only through the friction. The electron velocity $V_{0}$ is assumed nearly constant 
by the parallel electric field of the wave. From (77), assuming that the ions are initially at rest, the ion velocity, normalized to $V_{0}$, becomes $1-\exp \left(-\nu_{i e} t\right)$. Taking $n_{0}=10^{18} \mathrm{~m}^{-3}, T_{e}=10^{4} \mathrm{~K}, T_{i}=2 \cdot 10^{3} \mathrm{~K}$, we have $\nu_{i e}=7 \cdot 10^{3} \mathrm{~Hz}$. A simple plot of the ion velocity reveals that it becomes close to 1 already after about 0.0007 seconds.

b) Taking another extreme case where electrons initially, due to any external reason acquire a velocity $v_{e 0}=V_{0}$, without any additional force, and where $v_{i 0}=0$. In this case the electron velocity is not kept constant, the interaction of the two fluids yields the evolution of the two velocities:

$$
\begin{aligned}
& \vec{v}_{e}=\frac{\nu_{i e} \vec{V}_{0}}{\nu_{e i}+\nu_{i e}}+\frac{\nu_{e i} \vec{V}_{0}}{\nu_{e i}+\nu_{i e}} \cdot \exp \left[-\left(\nu_{e i}+\nu_{i e}\right) t\right], \\
& \vec{v}_{i}=\frac{\nu_{i e} \vec{V}_{0}}{\nu_{e i}+\nu_{i e}}-\frac{\nu_{i e} \vec{V}_{0}}{\nu_{e i}+\nu_{i e}} \cdot \exp \left[-\left(\nu_{e i}+\nu_{i e}\right) t\right] .
\end{aligned}
$$

Here, the electron and ion velocities monotonously change in time towards the common velocity (the first term on the right-hand side) $v_{c} \simeq V_{0} m_{e} / m_{i} \ll V_{0}$ which, for the same parameters as above, is achieved within the time interval shorter than $10^{-6}$ sec. The characteristic time for the velocity relaxation is $\sim 1 /\left(\nu_{e i}+\nu_{i e}\right)$. This is presented in Fig. 1.

A real physical situation, as in the case of the drift wave discussed earlier, is expected to be somewhere in between the two extremes presented above. Hence, in spite of a huge mass difference, the collisions (friction) will force ions to move along the magnetic field lines, and, due to the same reason, the electron velocity amplitude associated with the drift wave is expected to be considerably smaller.

VI. We shall check now the case of a plasma with dominant electron collisions with neutrals. The electron parallel momentum Eq. (6) now reads

$$
0=e n_{0} \frac{\partial \phi_{1}}{\partial z}-\kappa T_{e} \frac{\partial n_{1}}{\partial z}-m_{e} n_{0} \nu_{e n}\left(v_{e z 1}-v_{n z 1}\right)
$$

The ion dynamics is the same as above, so we use Eq. (18). The dynamics of neutrals is completely described by

$$
\frac{\partial v_{n z 1}}{\partial t}=-\nu_{n e}\left(v_{n z 1}-v_{e z 1}\right) .
$$


This is used in Eq. (23) , with the momentum conservation condition that now reads $m_{n} n_{n} \nu_{n e}=m_{e} n_{0} \nu_{e n}$, yielding

$$
v_{e z 1}=\frac{i a k_{z} v_{T e}^{2}}{\nu_{e n}}\left(\frac{e \phi_{1}}{\kappa T_{e}}-\frac{n_{1}}{n_{0}}\right), \quad a=1+\frac{i \epsilon \nu_{e n}}{\omega} .
$$

Here, $\epsilon=m_{e} n_{0} /\left(m_{n} n_{n}\right)$, and in view of Eq. (2) this is a small quantity for any plasma. For instance, for an electron-proton plasma in a hydrogen gas $m_{n}=m_{i}=m_{p}$, it is of the order $10^{-6}$ or less. Equation (25) is used in the electron continuity Eq. (3) yielding

$$
\frac{n_{1}}{n_{0}}=\frac{\omega_{*}+i a k_{z}^{2} D}{\omega+i a k_{z}^{2} D}, \quad D=\frac{v_{T e}^{2}}{\nu_{e n}} .
$$

In the case $\omega, \omega_{*} \ll k_{z}^{2} D$, Eq. (26) can be written as

$$
\frac{n_{1}}{n_{0}}=\left[1+\frac{\epsilon^{2} \nu_{e n}^{2}}{\omega^{2}}-\frac{\epsilon \nu_{e n}}{\omega} \frac{\omega+\omega_{*}}{k_{z}^{2} D}+\frac{i\left(\omega-\omega_{*}\right)}{k_{z}^{2} D}\right]\left(1+\frac{\epsilon^{2} \nu_{e n}^{2}}{\omega^{2}}-\frac{2 \epsilon \nu_{e n}}{k_{z}^{2} D}\right)^{-1} .
$$

We have $1 \gg 2 \epsilon \nu_{e n} / k_{z}^{2} D \gg \epsilon^{2} \nu_{e n}^{2} / \omega^{2}$, the real part is therefore very close to unity, while the imaginary part is simply $i\left(\omega-\omega_{*}\right) / k_{z}^{2} D$. Consequently, combining Eq. (27) with Eq. (8), the real part of the frequency appears described as earlier by Eq. (14), while the imaginary part becomes similar to Eq. (16), viz.

$$
\omega_{i} \simeq \frac{\omega_{r}^{2}}{k_{z}^{2} D} \frac{\omega_{r}^{2} k_{y}^{2} \rho_{s}^{2}-k_{z}^{2} c_{s}^{2}}{\omega_{r}^{2}\left(1+k_{y}^{2} \rho_{s}^{2}\right)+k_{z}^{2} c_{s}^{2}} .
$$

However, we stress again that Eq. (16) follows from a formally incorrect procedure.

Compared to the previously discussed $e-i$ collision case (20), it is seen that i) in both cases the threshold is caused by the ion sound response, however, ii) the instability threshold in Eq. (28) is shifted towards higher frequencies (because $k_{y} \rho_{s}$ is usually less than unity).

Although the two cases describe two physically different plasma environments, the explanation for case ii) should be as follows. The lower threshold frequency in the $e-i$ case implies that electrons experience a larger amount of collisions with ions within a wave period, which is in fact necessary to compensate for the ion movement in the parallel direction (due to the parallel electric field). This is because moving ions (in the same direction as electrons) represent a less efficient barrier for electron parallel motion and, in order to have the necessary phase shift between the density and potential, the electrons should have more collisions for the instability to take place. On the other hand, in the $e-n$ case, a higher frequency (equivalent to a smaller amount of collisions) for the instability 
to develop is possible because neutrals are less movable in the parallel direction (they do not react to the parallel electric field), and therefore they represent a more effective barrier.

VII. To conclude, the self-consistent inclusion of the momentum conservation in the ion and electron equations, which originates from the collisions between the two fluids, yields a different instability threshold that, to the best of our knowledge, has not been discussed in the literature so far. The correct expressions (20) and (28) should be used for estimates of the growth rate and the instability threshold for the collisional drift mode.

Acknowledgements: The results presented here are obtained in the framework of the projects G.0304.07 (FWO-Vlaanderen), C 90205 (Prodex 9), GOA/2004/01 (K.U.Leuven), and the Interuniversity Attraction Poles Programme - Belgian State - Belgian Science Policy. JV would like to thank N. D'Angelo for fruitful discussions. 


\section{References}

[1] J. Weiland, Collective Modes in Inhomogeneous Plasmas (Institute of Physics Pub., Bristol, 2000) p. 32.

[2] P. M. Bellan, Fundamentals of Plasma Physics (Cambridge Univ. Press, Cambridge, 2006) pp. 253-258.

[3] J. Vranjes and S. Poedts, Phys. Lett. A 348, 346 (2006).

[4] J. Vranjes, B. P. Pandey, and S. Poedts, Planet. Space Sci. 54, 695 (2006).

[5] R. J. Goldston and P. H. Rutherford, Introduction to Plasma Physics (Institute of Physics Pub., Bristol, 1995) p. 382.

[6] S. S. Moiseev and R. Z. Sagdeev, J. Exp. Theor. Phys. (U.S.S.R.) 44, 763 (1963).

[7] N. D’Angelo, Phys. Fluids 6, 592 (1963).

[8] N. D'Angelo and R. V. Motley, Phys. Fluids 6, 422 (1963).

[9] E. Gravier, X. Caron, G. Bonhomme, T. Pierre, and J. L. Briançon, Eur. Phys. J. D 8, 451 (2000).

[10] J. Vranjes, A. Okamoto, S. Yoshimura, S. Poedts, M. Kono, and M. Y. Tanaka, Phys. Rev. Lett. 89, 265002 (2002).

[11] A. Okamoto, K. Hara, K. Nagaoka et al., Phys. Plasmas 10, 2211 (2003).

[12] O. Grulke, F. Greiner, T. Klinger, and A. Piel, Plasma. Phys. Control. Fus. 43, 525 (2001).

[13] C. Riccardi and Å. Fredriksen, Phys. Plasmas 8, 199 (2001).

[14] Å. Fredriksen, C. Riccardi, L. Cartegni, and H. Pécseli, Plasma. Phys. Control. Fus. 45, $721(2003)$.

[15] A. A. Mamun and P. K. Shukla, Phys. Plasmas 7, 3499 (2000).

[16] W. X. Wang, Z. Lin, W. M. Tang et al. Phys. Plasmas 13, 092505 (2006). 
[17] G. M. Staebler, J. E. Minsey, and R. E. Waltz, Phys. Plasmas 14, 055909 (2007).

[18] M. Mitchner and C. H. Kruger, Partially Ionized Gasses (John Willey and Sons, New York, 1973,) p. 102.

[19] A. Zecca, G. P. Karwasz, and R. S. Brusa, Riv. Nuovo Cim. 19, 128 (1996).

[20] J. A. Ratcliffe, The Magneto-Ionic Theory and its Applications to the Ionosphere (Cambridge University Press, Cambridge, 1959) p. 33.

[21] J. Vranjes and S. Poedts, Phys. Plasmas 13, 122103 (2006). 


\section{Figure caption:}

Fig. 1. The evolution of the ion velocity from Eqs. (21, 22) for an electron-ion plasma, normalized to $\nu_{i e} V_{0} /\left(\nu_{e i}+\nu_{i e}\right)$, and for $v_{e 0}=V_{0}, v_{i 0}=0$. 


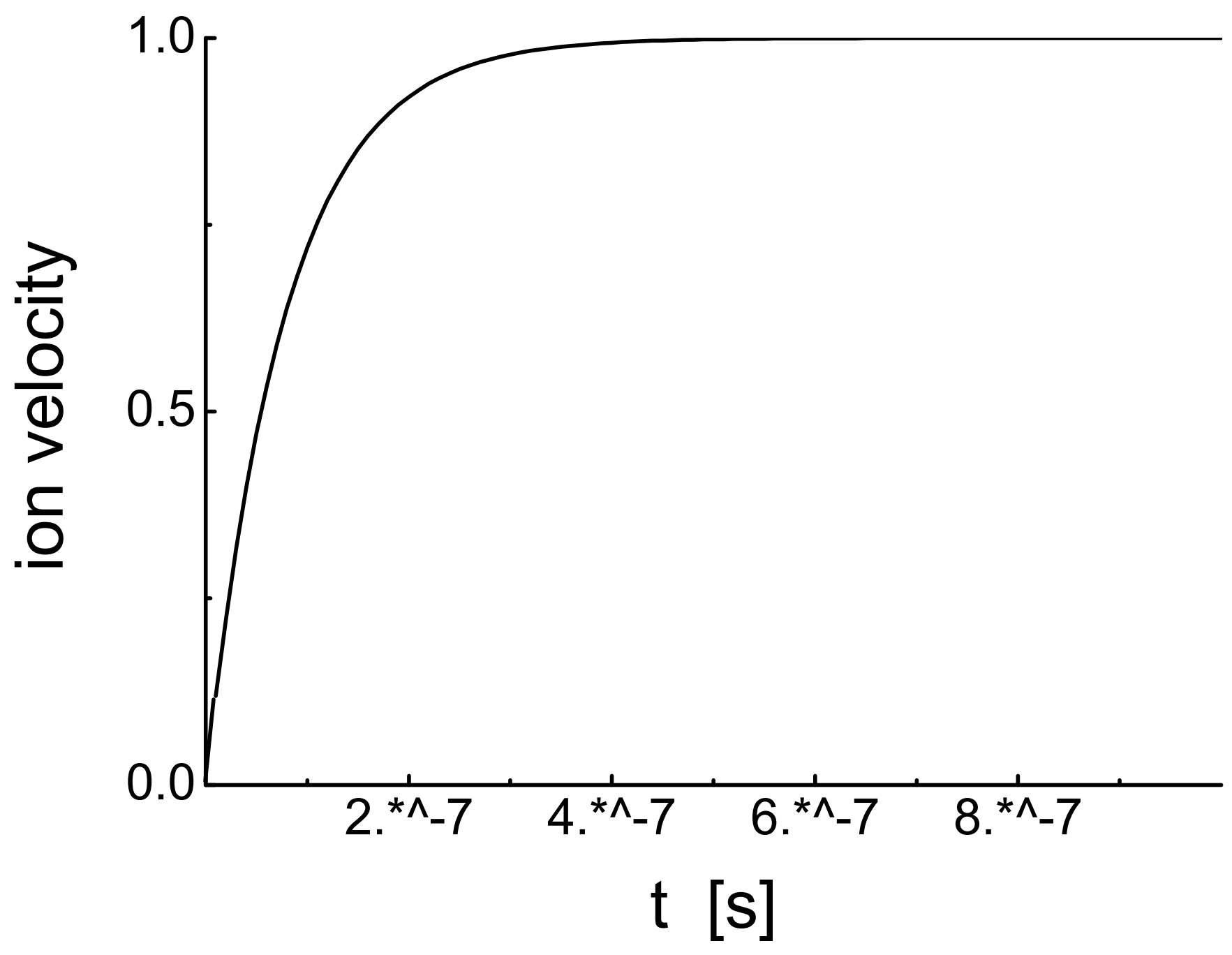

